\title{
Erratum to: Evaporative cooling and the Mpemba effect
}

\author{
M. Vynnycky $\cdot$ S. L. Mitchell $\cdot$ N. Maeno
}

Published online: 22 February 2011

(C) Springer-Verlag 2011

\section{Erratum to: Heat Mass Transfer (2010) 46:881-890 \\ DOI 10.1007/s00231-010-0637-z}

Due to an error in postprocessing, some results in the published paper are incorrect. For example, Fig. 9 suggests

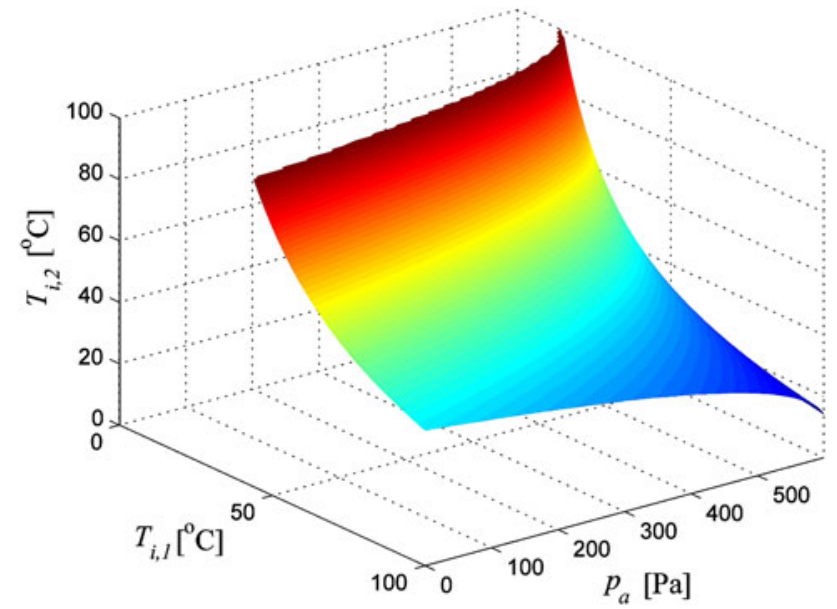

Fig. 1 All $\left(T_{i, 1}, T_{i, 2}, p_{a}\right)$-combinations above the surface lead to the Mpemba effect with respect to $t_{0}$. The surface is symmetric about $T_{i, 1}=T_{i, 2}$

The online version of the original article can be found under doi:10.1007/s00231-010-0637-z.

M. Vynnycky $(\bowtie) \cdot$ S. L. Mitchell

Mathematics Application Consortium for Science and Industry

(MACSI), Department of Mathematics and Statistics,

University of Limerick, Limerick, Republic of Ireland

e-mail: michael.vynnycky@ul.ie

\section{N. Maeno}

Professor Emeritus of Hokkaido University, Hanakawa Minami

7-2-133, Ishikari, Hokkaido 061-3207, Japan

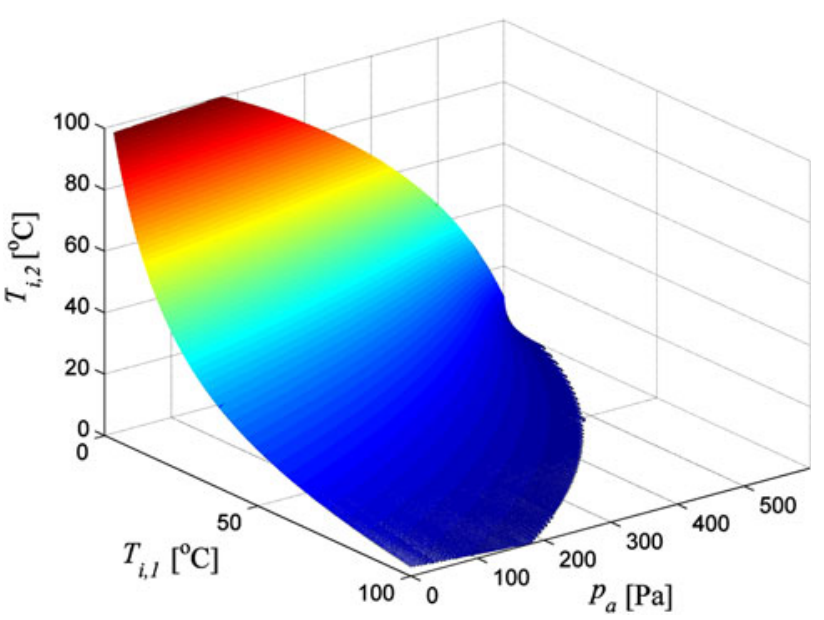

Fig. 2 All $\left(T_{i, 1}, T_{i, 2}, p_{a}\right)$-combinations above the surface lead to the Mpemba effect with respect to $t_{f}$. The surface is symmetric about $T_{i, 1}=T_{i, 2}$

that for $p_{a}=0 \mathrm{~Pa}$, it would be possible to see the Mpemba effect by performing one experiment for a value of $T_{i, 1}$ that is just below to $100^{\circ} \mathrm{C}$ and a second experiment for a value of $T_{i, 2}$ that is just above $0^{\circ} \mathrm{C}$. However, consideration of curve $a$ in Fig. 6 indicates that this combination will not give the Mpemba effect; instead, although perhaps not so distinct from Fig. 6, it is necessary to take $T_{i, 2}$ greater than approximately $44^{\circ} \mathrm{C}$. A similar type of error has occurred in Fig. 10. Therefore, we present here, in Figs. 1 and 2 respectively, the correct results for Figs. 9 and 10.

Acknowledgments M. Vynnycky and S. L. Mitchell acknowledge the support of the Mathematics Applications Consortium for Science and Industry (www.macsi.ul.ie) funded by the Science Foundation Ireland Mathematics Initiative Grant 06/MI/005. 